\title{
Concluding Remarks: Non-Electromagnetic Windows in Astrophysics
}

\author{
Catherine J. Cesarsky \\ European Southern Observatory, Karl-Schwarzschild-Str. 2, \\ D-85748 Garching, Germany
}

It is a pleasure, once again, to take part in a meeting dedicated to a host of exciting topics in high energy and particle astrophysics at an IAU General Assembly; my last time was at a meeting I had myself organized at the GA in Baltimore 15 years ago. In the mean time these subjects have attracted much interest among particle physicists, but had been receiving less attention from the general astrophysicists attending IAU GAs.

In those fifteen years, there has been an explosion of knowledge in astrophysics, due to simultaneous advances at virtually all the wavelengths of the electromagnetic spectrum. This led to breakthroughs in numerous fields such as cosmology, galaxy evolution and star formation. At the same time, extra solar planets have at long last been unambiguously discovered. In comparison, excluding neutrino discoveries, which led to a Nobel Prize in 2002, astrophysics from non electromagnetic windows appears to have progressed at a slower pace and to have attracted less attention. When so many important discoveries are at hand, or easy to get, why struggle and attempt more difficult searches?

At this Joint Discussion, we heard from the adventurous at heart, pioneers who are prepared to risk considerable parts of their career building experiments that aim at ambitious, but unassured objectives. And we also heard that the first or next generation of experiments is getting ready to go, so that the forthcoming years promise to be exciting in these exploratory fields too.

We heard first about the huge success of the solar neutrino experiments, which make it possible now to truly understand the source of the solar energy, and at the same time have extremely interesting implications on neutrino oscillations. This has naturally become a hot topic for particle physicists. The study of solar oscillations brings additional, crucial information of neutrino properties and of important stellar processes, convection and rotation.

Gamma ray astrophysics was not discussed here, but the results obtained in this spectral window are well connected to topics covered, such as cosmic ray acceleration and high-energy neutrinos, and give impressions of what can be obtained. We now have a reasonable understanding of the emission of the galaxy from the tens of $\mathrm{MeV}$ to the few $\mathrm{GeV}$ region, and even point source and diffuse emission in gamma ray lines has been reported; the Integral satellite will bring new and possibly decisive information on these topics. Ground Cerenkov experiments continue to monitor sources at higher energies, up to the several $\mathrm{TeV}$ range, detecting continuous sources and occasional outbursts, but not supernova remnants. The space mission GLAST, which will reach $300 \mathrm{GeV}$, will soon complement them. Bold ground experiments aiming at the detection of gamma rays at even higher energies have only established upper limits. The study of 
gamma ray bursts has advanced most in the last few years. It is now proven that their sources are at cosmological distances and that one class, at least, is due to hypernova explosions. In the future gamma ray bursts may be an excellent tool to study the universe at high redshifts, surpassing absorption studies in quasars if the optical or infrared afterglows can be caught sufficiently early.

The studies and measurements made until now have not indentified with certainty the sources of cosmic ray nuclei, even if inferences can be made about supernovae and/or OB associations, as we were reminded at this meeting. The direct detection of high-energy neutrinos would of course radically change the panorama. Predictions of the emissions expected according to various models of galactic sources of cosmic rays and of pulsars have been made and the experiments are forthcoming. With AMANDA, at least interesting limits on the putative galactic sources will be obtained in the near future, and eventually Ice Cube and ANTARES will explore also extragalactic space.

The new generation of gravitational wave detectors will have an equally adventurous task. While there is little doubt that gravitational waves pervade the universe, there is a wide range of predictions and a high chance of surprises. The measurements are extremely delicate, and I wish much luck to LIGO, and to V.IRGO, inaugurated in Italy during the Sydney IAU GA. After these exploratory ground experiments, which probably need enhancements to sensitivity, such as that planned for LIGO, to discover cosmic sources, the space mission LISA will open a whole new parameter space, with superior chances of success.

Even more adventurous and brave are the attempts at direct detection of dark matter. The density of dark matter in the universe as a whole, or in clusters of galaxies, is today better constrained than that of dark matter in the solar neighborhood. In any case, the various experiments available today do not appear yet to exhibit sensitivities that could be conducive to detection. But the progress is steady, and the stakes are very high. In the mean time, more about dark matter distribution and properties will be learned using classical astrophysical tools, optical and $\mathrm{X}$ ray telescopes, combined with models of weak shear and of the thermal behavior of intergalactic gas.

I left for the end the Ultra High Energy Cosmic Ray experiments. Having worked myself on cosmic rays for many years in the first part of my career, I am particularly excited by the opportunities offered by the forthcoming experiments in this field. Here again, generations of experiments have shown that cosmic rays' of energies as high as $10^{20} \mathrm{eV}$ exist, even if the upper limit in energy is under dispute. Small number statistics have not allowed a credible conclusion on the distribution or distance of the sources to be reached, a situation quite similar to that of gamma ray bursts before the Compton Gamma Ray Observatory. The Auger collaboration, especially if it succeeds in building the northern as well as the southern part of the experiment, should for the first time obtain results with sufficient statistical weight (see also, later, the Space Station mission EUSO for real UHE Cosmic Ray astronomy!). It is intriguing to think that we may have a better chance of discovering the sources of UHECR than those of the bulk of the particles in the $\mathrm{GeV}$ range!

In conclusion: this was a very interesting Joint Discussion, and I hope to hear more about these topics and about the results of the new experiments, at the next IAU GA in Prague. 